\title{
The Spatial QRS-T Angle and the Spatial Ventricular Gradient: Normal Limits for Young Adults
}

\author{
RWC Scherptong, SC Man, S Le Cessie, HW Vliegen, HHM Draisma, \\ AC Maan, MJ Schalij, CA Swenne \\ Leiden University Medical Center, Leiden, the Netherlands
}

\begin{abstract}
We computed normal limits of the spatial QRS-T angle $(S A)$ and the spatial ventricular gradient $(S V G)$ in 660 normal resting ECGs (449 female / 211 male) recorded in healthy subjects aged 18-29 years. Values for males and females were compared, and normal limits for males from this study were compared to the normal limits for males, published previously in 1967.

In females, the $S A$ was sharper (females: $66 \pm 23^{\circ}$, males: $\left.80 \pm 24^{\circ}, P<0.001\right)$ and the $S V G$ magnitude was smaller (females: $81 \pm 23 \mathrm{mV} \cdot \mathrm{ms}$; males: $110 \pm 29$ $m V \cdot m s, P<0.001)$ than in males. The $S V G$ magnitude in males was larger than that published in $1967(79 \pm 28$ $m V \cdot m s ; P<0.001)$.

$S A$ and $S V G$ depend strongly on gender. The newly calculated SVG magnitude in males differs strikingly from the 1967 value; explanations for this difference are given.
\end{abstract}

\section{Introduction}

The spatial QRS-T angle (SA) and the spatial ventricular gradient (SVG) are classical electrocardiographic parameters that provide information on cardiac conduction system functioning and on ventricular action potential duration heterogeneity[1;2]. These parameters are typically calculated in the vectorcardiogram (VCG), either directly recorded by the 8electrode Frank lead system, or by synthesizing a VCG from a standard 10-electrode 12-lead electrocardiogram (ECG) by a matrix operation[3;4].

The SA is the angle between the spatial orientations of the QRS- and the T axes. Normally, the orientation of the depolarization axis and repolarization axis is in a similar direction[5]. This results in a sharp SA, which corresponds to a predominantly concordant ECG. When pathological changes occur, the ECG becomes more discordant and the SA widens[6]. Recently, Kardys et al. demonstrated that, in the general population, an SA wider than 105 degrees[2], was associated with a higher risk for cardiovascular death. Therefore the SA is regarded useful in cardiac risk assessment.

The SVG is defined as the vectorial QRST integral. Unlike most other ECG parameters, the SVG is not influenced by changes in ventricular conduction pattern; it only changes if the distribution of the ventricular action potential morphology and/or duration is altered[1]. Therefore, the SVG is a valuable tool to discriminate primary (as a consequence of a change in the action potential) from secondary (as a consequence of a change in conduction) $\mathrm{T}$ wave phenomena.

Until now, the first and only normal limits for SA and SVG were calculated by Pipberger and colleagues in 1964 and 1967[7;8]. Their study group consisted of hospitalized men in an age range of 19-84 years and without a history of cardiovascular disease. Our current study was prompted by a number of reasons:

Female subjects have to be studied (see the differences in male-female SVG values in Yamauchi et al.[9]);

Normal subjects should not be hospitalized;

Minnesota criteria for normality[10] should be applied more rigidly than in the 1964 and 1967 studies;

Normal limits have to be calculated in VCGs that are synthesized from regular 12-lead ECGs, as this is currently common technology.

Therefore, the aim of our study was to determine normal limits of the SA and the SVG magnitude and orientation, as derived from synthesized VCGs of healthy young adult males and females, and to compare these normal limits to those previously published.

\section{Methods}

\subsection{Subjects}

Standard 10-second 12-lead ECGs were obtained from medical students. Length and weight were measured, body mass index (BMI) was calculated, and body surface area (BSA) was obtained using Mosteller's formula[11]. 
Normality of ECGs was confirmed with the Minnesota ECG coding protocol[10]. Normal ECGs were included when subjects fulfilled the following age and heart rate criteria: 18 yrs - 29 yrs; 50 beats per minute (bpm) - 100 bpm (Minnesota criterion 8-7, 8-8).

\subsection{Electrocardiographic analysis}

ECGs were analyzed with the MATLAB-based (The MathWorks, Natick, USA) computer program LEADS (Leiden ECG Analysis and Decomposition Software)[12]. LEADS first detects all QRS complexes and corrects the baseline. Then, supervised beat selection for subsequent averaging is done; acceptation/rejection of beats is based on signal-to-noise ratio, on interbeat interval regularity and on representative QRS-T morphology. After computation of the averaged beat, an averaged vectorcardiographic beat is synthesized using the inverse Dower matrix [3;4]. In this averaged beat, the onset of the QRS complex, the J point and the end of the $\mathrm{T}$ wave are detected automatically. The default position of the J point can be adjusted manually with a crosshaircursor procedure, facilitating accurate placement according to the Minnesota ECG coding protocol. Global end of $\mathrm{T}$ is calculated in the vector magnitude signal as the intersection of the steepest tangent to the descending limb of the $\mathrm{T}$ wave and the base-line. Given these landmarks in time, the SA as well as the SVG azimuth, elevation and magnitude are computed. Azimuth and elevation are represented in accordance with the AHA vectorcardiography coordinate Standard[13].

\subsection{Statistical analysis}

SPSS (12.0.1, SPSS Inc., USA) was used for statistical analysis. Data are reported as mean with standard deviation (SD). Unpaired Student t-tests were used to compare values of males and females. The Spearman rank correlation was calculated between the anthropomorphic measures (height, weight, BMI, BSA) and the SA and SVG. Normal limits were set at the 2nd and 98th percentile[7]. We used logarithmically transformed linear regression to calculate normal limits for SVG magnitudes depending on heart rate. A Student t-test was used to compare our normal limits of the SA and SV to the earlier published normal limits[7;8]. P values $<0.05$ were considered significant.

\section{Results}

\subsection{Normal limits}

ECGs were taken in 804 subjects. The ECGs of 67 subjects were excluded because of technical reasons (electrode displacement, missing leads, signal noise), 22
ECGs were considered abnormal according to the Minnesota criteria. In 41 subjects heart rate criteria, and in 14 subjects age criteria were not met. This left the ECGs of 660 (449 female, 211 male) subjects for analysis.

Table 1 summarizes the descriptive statistics of SA and SVG and the normal limits in the form of the $2^{\text {nd }}$ and $98^{\text {th }}$ percentile, which can be visually appreciated in Figure 1. All mean values of males differed significantly from the mean values of female subjects. Male subjects had significantly wider spatial angles and larger SVG magnitudes as compared to female subjects. Furthermore, the SVG orientation in males was more anterior and slightly more superior than in female subjects.

Table 1. Normal limits of SA and SVG

\begin{tabular}{lllll}
\hline \hline & \multicolumn{2}{l}{ Females } & Males \\
& Mean & Limits & Mean & Limits \\
\hline SA & $66^{*}$ & $\mathbf{2 0 - 1 1 6}$ & $80^{*}$ & $\mathbf{3 0 - 1 3 0}$ \\
SVG magnitude & $81^{*}$ & $\mathbf{3 9 - 1 4 3}$ & $110^{*}$ & $\mathbf{5 9 - 1 8 7}$ \\
SVG azimuth & $-13^{*}$ & $\mathbf{- 3 8 - 2 0}$ & $-23^{*}$ & $\mathbf{- 5 2 - 1 3}$ \\
SVG elevation & $30^{*}$ & $\mathbf{1 2 - 4 8}$ & $27^{*}$ & $\mathbf{8 - 4 7}$ \\
\hline \hline
\end{tabular}

A prominent correlation of $-0.36(\mathrm{P}<0.01)$ in females and $-0.46(\mathrm{P}<0.01)$ in males was found between SVG magnitude and heart rate. Lower heart rates were associated with larger SVG magnitudes than higher heart rates. Also, the distribution of the SVG magnitudes was wider for lower heart rates than for higher heart rates. Therefore, SVG magnitudes were logarithmically transformed after which a linear regression of log (SVGmagnitude) on heart rate (HR) was made. The following regression equations were found:

Females:

${ }^{10} \log \left(\mathrm{SVG}_{\text {magnitude }}\right)=2.18-3.95 * 10^{-3} \cdot \mathrm{HR}(\mathrm{SD}=0.12)$

Males:

${ }^{10} \log \left(\mathrm{SVG}_{\text {magnitude }}\right)=2.38-4.89 * 10^{-3} \cdot \mathrm{HR}(\mathrm{SD}=0.11)$

\subsection{Earlier published normal limits}

In Figure 1, both our results and the earlier published results of the Pipberger group[7;8] are depicted. When comparing the results of the Pipberger group with the results of our male subjects, the most striking differences 
in normal limits are found in the ventricular gradient magnitude and the ventricular gradient elevation upper limits. Our values for the ventricular gradient magnitude are $47 \mathrm{mV} \cdot \mathrm{ms}$ larger and our elevation is $16^{\circ}$ smaller. Moreover, differences of the same order of magnitude and direction are seen in the mean values of these parameters $\left(31 \mathrm{mV} \cdot \mathrm{ms}\right.$ and $9^{\circ}$, respectively; $\left.P<0.01\right)$.
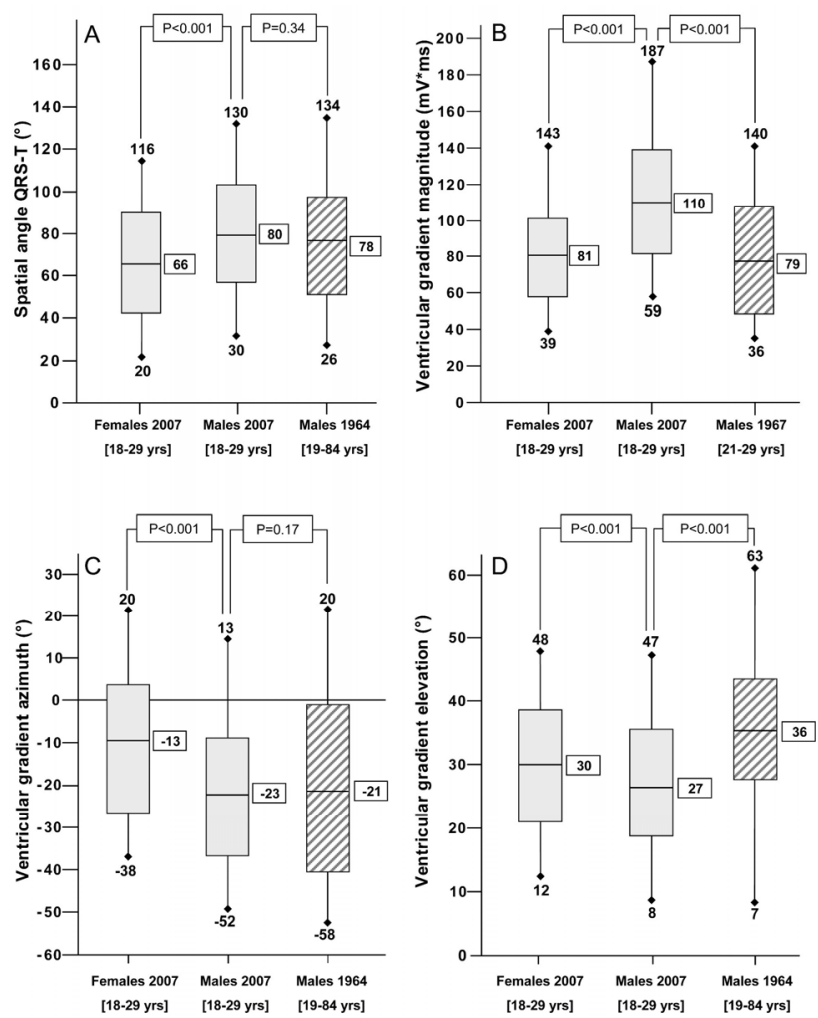

Figure 1. Mean, standard deviation, and normal limits of SA and SVG. Grey boxes: results from our study. Diagonally marked boxes: results from the studies by Draper et al.[7] (panels A, C, D) and by Pipberger et al.[8] (panel B).

\section{Discussion and conclusions}

In the present study, we measured normal limits of the spatial QRS-T angle and the spatial ventricular gradient in young healthy adults. Key findings were that all values of male and female subjects differed significantly, thus underscoring the need for separate normal limits. There was a striking influence of heart rate on the spatial ventricular gradient magnitude. Comparison of the results of our study with earlier published results demonstrated prominent differences in normal limits of the spatial ventricular gradient magnitude and elevation.

SA and SVG both strongly depended on gender. In our study, males had a larger SA and SVG magnitude than females. The most important gender-dependent difference in SVG orientation was found in the SVG azimuth, which was directed more anteriorly in males than in females. The difference in SVG elevation between males and females, albeit significant, was small $\left(3^{\circ}\right)$.

In the present study, mean SA was $80^{\circ}$ for males and $66^{\circ}$ for females. In a study by Rautaharju et al.[14], in which mortality risk was investigated in a large $(n=4,912)$ unselected group of older subjects (ages $>65 \mathrm{yrs}$ ), similar differences between males and females for the SA were found. They reported a mean SA of $81^{\circ}$ for males $(72.8 \pm$ $5.7 \mathrm{yrs})$ and a mean SA of $67^{\circ}$ for females $(72.2 \pm 5.3$ yrs). In another study, gender related differences in SVG magnitude were investigated in a small young (ages 2030 yrs) group of 30 male and 30 female Japanese subjects by Yamauchi and colleagues[9]. They found similar differences between SVG magnitude in males (105 $\mathrm{mV} \cdot \mathrm{ms}$ vs. $110 \mathrm{mV} \cdot \mathrm{ms}$ in our study) and females (81 $\mathrm{mV} \cdot \mathrm{ms}$, vs. $81 \mathrm{mV} \cdot \mathrm{ms}$ in our study).

It is unclear where the difference between males and females exactly originates. In the present study; weight, height and the derived parameters BMI and BSA were only weakly correlated to SVG and the SA. Therefore, these parameters cannot explain the observed malefemale differences. Possibly, part of the explanation is to be found in a different ratio between thorax dimensions and heart size, a different amount of subcutaneous fat, and presence of breast adipose tissue. Furthermore, parameters that relate to the difference in cardiac morphology between males and females (e.g., ventricular mass, wall thickness, electrophysiological characteristics) may further explain the difference in SA and SVG magnitude and orientation[15;16].

The studies by Pipberger and associates[7;8] are the only ones in which data on the SA and SVG are fully reported (mean, SD, $2^{\text {nd }}$ and $98^{\text {th }}$ percentile), which enables comparison to our normal limits (Figure 1). We found striking differences in both normal limits and means of the SVG magnitude and SVG elevation.

Diversity in the composition of the study groups (Pipberger et al. vs. the male subjects in our study group) and/or a methodological difference may underlie these differences in mean values and normal limits.

Firstly, the study group of Pipberger and colleagues consisted of hospitalized men (although without evidence for cardiovascular disease). However, also non-cardiac disease or the administration of non-cardiac medication can induce changes in cardiac electrophysiology that can result in (temporary) changes of the SA and/or SVG[17]. Secondly, Pipberger and associates used 8-electrode, 3lead Frank VCGs instead of 10-electrode, 12-lead standard ECGs and a synthesized VCG which nowadays is mostly used[2;14]. Obviously, this affects the shape of 
the vector loop and, consequently, may influence SA and/or SVG. Finally, in our study group, an important correlation was found between (resting) heart rate and SVG magnitude in both males and females (higher heart rates were associated with lower SVG magnitudes). This observation reflects directly the well-known electrophysiological phenomenon that the electrical heterogeneity in the ventricles increases with increasing intervals between heart beats[18]. In addition, this may provide an explanation for the difference in SVG magnitude between our study and the study by the Pipberger group. The exclusion boundary for lower heart rates in the Pipberger study group was $60 \mathrm{bpm}$, and not $50 \mathrm{bpm}$ as in our study group. Hence, large ventricular gradient magnitudes were partially filtered out by exclusion of low heart rates. Furthermore, as the study group of Pipberger and colleagues consisted of hospitalized subjects, resting heart rates may have been higher as compared to our non-hospitalized subjects leading to smaller SVG magnitudes in their population. Unfortunately, heart rate statistics were not reported by the Pipberger group, hence we cannot verify this possible explanation.

Recently, the SA was described as a risk stratifier for cardiovascular death in a study by Kardys et al.[2] and by Yamazaki and colleagues[19]. In these studies, SA in the low risk group was defined below $105^{\circ}$ and $100^{\circ}$, respectively. However, our study demonstrates that the normal SA can range up to $116^{\circ}$ in females and $130^{\circ}$ in males, indicating that current risk stratification criteria for the occurrence of cardiovascular death may be too strict, and should have been different for males and females.

Another, general, implication of our study is that normal limits of vectorcardiographic parameters should preferably be determined via the synthesized VCG technique, that rests on the now generally accepted standard 12-lead ECG instead of the Frank VCG recording protocol.

\section{References}

[1] Draisma HHM, Schalij MJ et al.: Elucidation of the spatial ventricular gradient and its link with dispersion of repolarization. Heart Rhythm. 2006;3:1092-9.

[2] Kardys I, Kors JA et al.: Spatial QRS-T angle predicts cardiac death in a general population. Eur Heart J. 2003;24:1357-64.

[3] Kors JA, van Herpen G et al.: Reconstruction of the Frank vectorcardiogram from standard electrocardiographic leads: diagnostic comparison of different methods. Eur Heart J. 1990;11:1083-92.
[4] Dower GE, Machado HB et al.: On deriving the electrocardiogram from vectoradiographic leads. Clin Cardiol. 1980;3:87-95.

[5] Durrer D, van Dam RT et al.: Total excitation of the isolated human heart. Circulation. 1970;41:899-912.

[6] van Huysduynen BH, Swenne CA et al.: Dispersion of repolarization in cardiac resynchronization therapy. Heart Rhythm. 2005;2:1286-93.

[7] Draper HW, Peffer CJ et al.: The Corrected Orthogonal Electrocardiogram and Vectorcardiogram in 510 Normal Men (Frank Lead System). Circulation. 1964;30:853-64.

[8] Pipberger HV, Goldman MJ et al.: Correlations of the orthogonal electrocardiogram and vectorcardiogram with consitutional variables in 518 normal men. Circulation. 1967;35:536-51.

[9] Yamauchi K, Sotobata I: Sex and age differences in ventricular gradient. Jpn J Med. 1991;30:504-8.

[10] Blackburn H, Keys A et al.: The electrocardiogram in population studies. A classification system. Circulation. 1960;21:1160-75.

[11] Mosteller RD: Simplified calculation of body-surface area N Engl J Med. 1987;317:1098.

[12] Draisma HHM, Swenne CA et al.: LEADS: An Interactive Research Oriented ECG/VCG Analysis System. Computers in Cardiology. 2005;32:515-8.

[13] Report of committee on electrocardiography, American Heart Association. Recommendations for standardization of leads and of specifications for instruments in electrocardiography and vectorcardiography: Circulation. 1967;35:583-602.

[14] Rautaharju PM, Ge S et al.: Comparison of mortality risk for electrocardiographic abnormalities in men and women with and without coronary heart disease (from the Cardiovascular Health Study). Am J Cardiol. 2006;97:30915.

[15] Surawicz B, Parikh SR: Differences between ventricular repolarization in men and women: description, mechanism and implications. Ann Noninvasive Electrocardiol. 2003;8:333-40.

[16] Sotobata I, Richman H et al.: Sex differences in the vectorcardiogram. Circulation. 1968;37:438-48.

[17] Albrecht CA: Proarrhythmia with non-antiarrhythmics. A review. Cardiology. 2004;102:122-39.

[18] Antzelevitch C, Fish J: Electrical heterogeneity within the ventricular wall. Basic Res Cardiol. 2001;96:517-27.

[19] Yamazaki T, Froelicher VF et al.: Spatial QRS-T angle predicts cardiac death in a clinical population. Heart Rhythm. 2005;2:73-8.

Address for correspondence

Cees A Swenne

Leiden University Medical Center

Department of Cardiology, C5-P

PO Box 9600

2300 RC Leiden, The Netherlands

E-mail: C.A.Swenne@LUMC.nl 